\title{
New Approaches to Extending the Performance of Brillouin Based Slow Light Systems
}

\author{
C. Jáuregui, P. Petropoulos and D.J. Richardson \\ Optoelectronics Research Centre, University of Southampton, \\ Southampton SO17 1BJ, United Kingdom
}

\begin{abstract}
We review the development of several new approaches for extending the performance of Brillouin based slow light systems. In particular we describe the use of cavity effects to enhance the achievable delays, gain saturation to decouple the delay and associated signal gain, and the use of tailored pump beams to effect reshaping and retiming of periodic signals.
\end{abstract}

\section{INTRODUCTION}

Brillouin-assisted slow light has received much attention since its first demonstrations in 2005 [1, 2]. As well as being of inherent physical interest, these early experiments, carried out in a conventional fiber at room temperature, seemed to point to a possible route to the development of several important all-optical devices ranging from simple all optical tuneable delay lines through to optical buffers.

Much of the subsequent research effort has targeted, in one way or another, the pursuit of these important applications. As a consequence many of the factors limiting the technique have already been overcome. For example, ways to enhance the bandwidth of the delayed signals by broadening the Brillouin gain profile [3, 4] have been developed, and the use of extreme nonlinearity non-silica fibers has been shown to provide a way to reduce the pump power and inherent device latency, as well as to increase the effective reduction in speed $[5,6]$. However, we and others, have also sought to gain a fuller understanding of the Brillouin delay mechanism, and to investigate ways of better exploiting it within more complex optical systems to enhance the gain-delay characteristics and functionality of slow light systems.

In this paper we review our work on the use of cavity effects to enhance the achievable delays, gain saturation to decouple the delay and associated signal gain, and the use of tailored pump beams to effect reshaping and retiming of periodic signals.

\section{CAVITY EFFECTS}

Taking into account that the dynamics of Brillouin scattering are strongly modified in the presence of external feedback [7], we investigated ways to employ an external cavity to enhance slow-light generation. The idea is that if the Brillouin peak coincides with one of the transmission maxima of the cavity, then constructive interference will take place leading to a sharpening of the gain peak. This process simultaneously narrows and enhances the gain peak, which ultimately gives rise to a reinforcement of the slow light effect that can provide delays even $100 \%$ higher than without the cavity [8]. We demonstrated this point in a slow-light system comprising a 2-m long bismuth oxide highly nonlinear fiber. The high core index creates a Fresnel reflectivity at the uncoated end facets of $\sim 2.5 \%$ when coupled to a SMF fiber. This gives rise to a low-finesse Fabry-Perot cavity enclosing the highly nonlinear fiber. We measured a maximum delay of $\sim 70 \mathrm{~ns}$ for 200ns Gaussian pulses using just $\sim 500 \mathrm{~mW}$ pump power, as shown in Fig.1. Without the presence of the cavity our simulations predict a delay of $\sim 30 \mathrm{~ns}$.

On the other hand, if the gain peak sits at one of the transmission minima the gain peak is broadened, which ultimately results in a smaller delay for the same pump power. This way the detuning of the gain peak relative to the transmission maxima of the cavity offers an additional means of control for the delay without changing the pump power.

It should be noted that although demonstrated in the context of Brillouin-assisted slow light, this cavity enhancement effect should work with any other gainassisted slow light technique.Ultimately the slow-light enhancement takes place due to a tighter fitting of the gain bandwidth to the signal bandwidth. Therefore, this effect can be most useful whenever there is some spare gain bandwidth (e.g. when using Raman scattering).

One requirement of the technique is the existence of intrapulse interference, which limits the maximum length of the cavity. Other cavity configurations, like a ring cavity, offer better performance for shorter lengths. However, even for this higher performance configuration, the cavity length required for high speed signals would be too short to use optical fibers, and other slow light media might be required (e.g. semiconductor devices).

\section{USE OF GAIN SATURATION}

One of the characteristics of gain-assisted slow light systems is that once the slow light medium has been chosen, there is a coupling between gain and delay, i.e. certain gain always provides a certain amount of delay. However, this can pose problems in real systems since signals with different delays would have considerably different amplitudes. Therefore, it would be desirable to decouple these two magnitudes. We have proposed a way to achieve this by working in the pump depletion regime and by using a variable optical attenuator (VOA). Since all the delay 


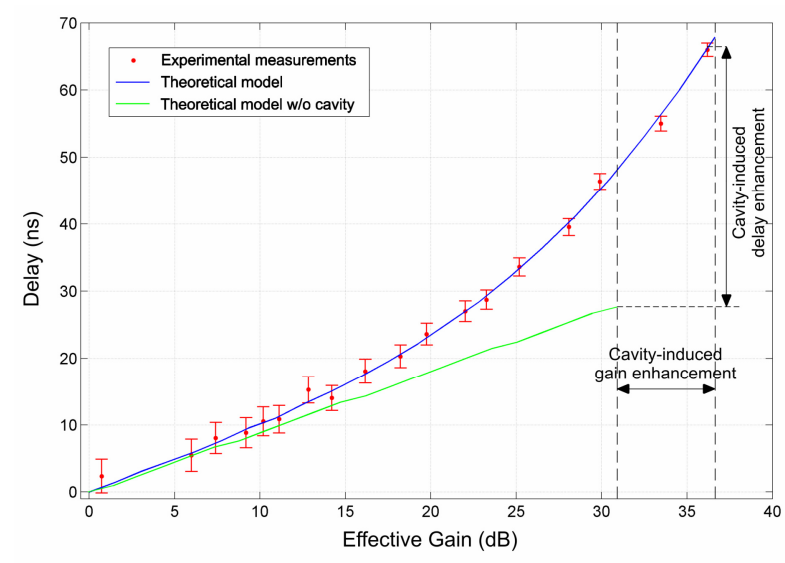

Fig.1. Experimental demonstration of slow light enhancement due to the presence of reflections. For comparison the theoretical prediction of the achievable delay without a cavity is also presented.

range offered by the fiber is swept in the pump depletion regime, the attenuation of the delayed probe under this operating condition provides access to shaded areas $A+B$ in Fig.2 (i.e. any combination of delay/gain offered by the system), whereas only the shaded area A would be accessible if operating in the linear regime. Alternatively, the operation in the pump depletion regime can be useful to obtain more equalized delayed signals or even signal with different gain levels but very similar delays (around the peak of Fig.2).

As can be seen in Fig.2, the pump depletion regime is characterized by a rapid decrease in the delay with increasing gain. The decrease of induced delay is due to pulse reshaping instead of any phase phenomenon. In spite of this, we have seen in our experiments that the amount of pulse distortion induced is very little provided the probe pulses are smooth and the delay is always kept $>0 \mathrm{~ns}$ [9].

\section{USE OF COMPLEX PUMP FIELDS}

Several works have attempted to broaden the narrow bandwidth of Brillouin gain, in order to make the effect applicable to telecoms signals. For example, in [3, 4] the pump was modulated by a pseudorandom bit sequence (PRBS) to uniformly broaden its bandwidth. By contrast to the continuous spectrum of a PRBS signal, a complex periodic pulse pattern exhibits a multiline spectrum that can be employed to generate a Brillouin gain comb. When appropriately handled, this process can be used to manipulate the individual harmonics of a high-speed periodic signal. This constitutes a very flexible and powerful way of signal processing that can be used for signal reshaping and/or retiming with very little gain change. It is important to remark that this technique is essentially independent of the frequency of the periodic signal.

\section{CONCLUSIONS}

We have shown that gain-assisted slow light, and particularly Brillouin-assisted slow light, can be strongly influenced by elements outside the Brillouin medium. These dependencies and the modification in behavior that they

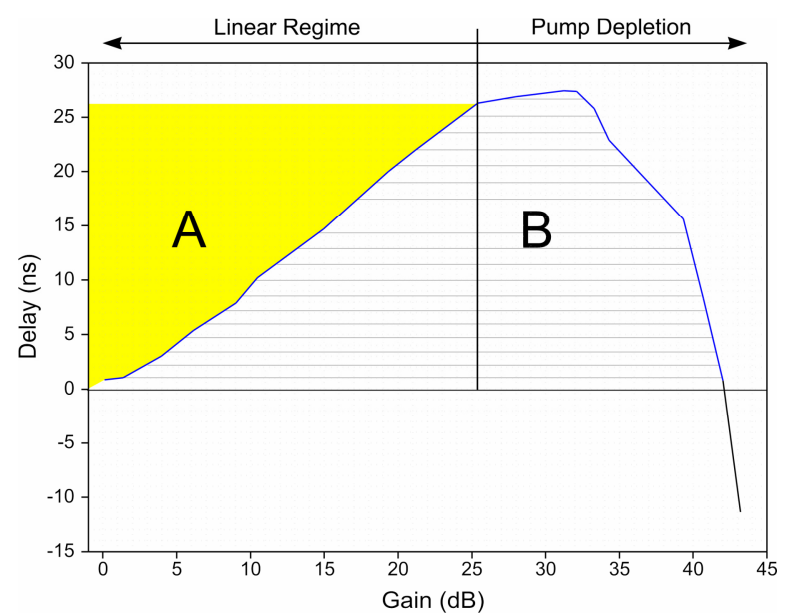

Fig.2. Delay vs. gain in a Brillouin-assisted slow-light system. The linear and pump depletion operation regimes are distinguished. Shaded area A illustrates the delay/gain combinations accessible by using an optical attenuator and operating in the linear regime. Operation in the pump depletion regime grants access to shaded areas $\mathrm{A}+\mathrm{B}$.

allow can be exploited in many different ways. We have presented some examples of how Brillouin-assisted systems can be engineered to enhance their functionality, such as the enhancement of the delay by using Brillouin-effects in a cavity, the decoupling of delay and gain, or the reshaping of signals by individual control of the their harmonics.

\section{ACKNOWLEDGMENT}

The authors acknowledge F. Koizumi and N. Sugimoto of Asahi Glass Company, Japan, for providing the Bi-HNLF. Also Furukawa Electric is thanked for providing the highlynonlinear silica fiber. C. Jáuregui also acknowledges the Fundación Ramón Areces for financial support.

\section{REFERENCES}

[1] M. González Herráez, K.Y. Song and L. Thévenaz, "Optically controlled slow and fast light in optical fibers using stimulated Brillouin scattering," Appl. Phys. Lett,.vol. 87, 081113, August 2005.

[2] Y. Okawachi, M.S. Bigelow, J.E. Sharping, Z. Zhu, A. Schweinsberg, D.J. Gauthier, R.W. Boyd and A.L. Gaeta, "Tunable all-optical delays via Brillouin slow light in an optical fiber," Phys. Rev. Lett., vol. 94, 153902, April 2005.

[3] M. González Herráez, K.Y. Song, and L. Thévenaz, "Arbitrarybandwidth Brillouin slow light in optical fibers," Optics Express, vol. 14, 1395-1400, February 2006.

[4] Z. Zhu, A.M.C. Dawes, D.J. Gauthier, L. Zhang and A.E. Willner, "12GHz-Bandwidth SBS slow light in optical fibers," presented at OFC 2006, Anaheim, California, 5-10 March. 2006, paper PDP1 (Postdeadline).

[5] C. Jáuregui, P. Petropoulos, and D.J. Richardson, "Slowing of pulses to c/10 with sub-watt power levels and low latency using Brillouin amplification in a bismuth oxide optical fiber," J. Lightwave Technol., vol. 25, pp. 216-221, January 2007.

[6] K.Y. Song, K.S. Abedin, K. Hotate, M. González Herráez and L. Thévenaz, "Highly efficient Brillouin slow and fast light using $\mathrm{As}_{2} \mathrm{Se}_{3}$ chalcogenide fiber," Optics Express, vol. 14, pp. 5860-5864, June 2006.

[7] A.L. Gaeta and R.W. Boyd, "Stimulated Brillouin scattering in the presence of external feedback," Int. J. Nonlinear Opt. Phys., vol. 1, pp. 581-594, 1992.

[8] C. Jáuregui, P. Petropoulos, and D.J. Richardson, "Brillouin assisted slow-light enhancement via Fabry-Perot cavity effects", Optics Express, vol. 15, pp.5126-5135, April 2007.

[9] C. Jáuregui, P. Petropoulos, and D.J. Richardson, "Delay-Gain Decoupling in Brillouin-Assisted Slow Light", to be published.. 